PROCEEDINGS OF THE

AMERICAN MATHEMATICAL SOCIETY

Volume 135, Number 11, November 2007, Pages 3723-3731

S 0002-9939(07)08871-5

Article electronically published on June 22, 2007

\title{
TURING INCOMPARABILITY IN SCOTT SETS
}

\author{
ANTONÍN KUČERA AND THEODORE A. SLAMAN
}

(Communicated by Julia Knight)

Abstract. For every Scott set $\mathcal{F}$ and every nonrecursive set $X$ in $\mathcal{F}$, there is a $Y \in \mathcal{F}$ such that $X$ and $Y$ are Turing incomparable.

\section{INTRODUCTION}

H. Friedman and A. McAllister posed the question whether for every nonrecursive set $X$ of a Scott set $\mathcal{F}$ there is a $Y \in \mathcal{F}$ such that $X$ and $Y$ are Turing incomparable (see Problem 3.2 and also Problem 3.3 in Cenzer and Jockusch [2]). We present a positive solution to the question, using recent results in the area of algorithmic randomness and also results on $\Pi_{1}^{0}$ classes.

1.1. Background and notation. We begin by discussing the background of the Friedman-McAllister question. We then review some basic definitions and establish our notational conventions.

1.1.1. Background. We let $2^{\omega}$ denote the set of infinite binary sequences. One can equivalently think of $2^{\omega}$ as the Cantor set. A finite binary sequence $\sigma$ determines an open neighborhood in $2^{\omega}$ by taking the set of all infinite extensions of $\sigma$. A binary tree $T$ determines a closed subset of $2^{\omega}$ by taking the complement of the union of open neighborhoods given by the elements of $T$ that have no extensions in $T$.

As is well known, the Cantor set is a canonical example of a compact set. This fact translates to binary trees in the form of König's Lemma that every infinite binary tree has an infinite path. However, the proof of König's Lemma is not computational. Not every infinite recursive binary tree has an infinite recursive path. Thus, the set of recursive reals does not verify the compactness of $2^{\omega}$ with respect to recursive closed sets (also called $\Pi_{1}^{0}$ classes). In order to study the consequences of compactness, we need richer subsets of $2^{\omega}$.

Definition 1.1. A Scott set is a nonempty set $\mathcal{F} \subseteq 2^{\omega}$ such that if $T \subseteq 2^{<\omega}$ is an infinite tree recursive in a finite join of elements of $\mathcal{F}$, then there is an infinite path through $T$ in $\mathcal{F}$.

Received by the editors February 20, 2006 and, in revised form, August 14, 2006.

2000 Mathematics Subject Classification. Primary 03D28.

Key words and phrases. Scott set, Turing degree, $K$-trivial, low for random.

The first author was partially supported by the Research Project of the Ministry of Education of the Czech Republic MSM0021620838.

The second author was partially supported by NSF grant DMS-0501167.

(C)2007 American Mathematical Society Reverts to public domain 28 years from publication 
Scott [20] proved that the sets representable in a complete extension of Peano Arithmetic form a Scott set. Scott sets occur naturally in the study of models of arithmetic. They are the $\omega$-models of $W K L_{0}$, an axiomatic treatment of compactness. In other words, they are the models of $W K L_{0}$ in which the natural numbers are standard.

One can test the power of compactness arguments by examining what is true in every Scott set. A natural family of questions comes from considering the Turing degrees represented in an arbitrary Scott set. For example, it is not merely the case that every Scott set has nonrecursive elements. Jockusch and Soare 8 ] showed that for any Scott set $S$ and any finite partial order $P$, there are elements of $S$ whose Turing degrees are ordered isomorphically to $P$. Thus, the existential theory of the Turing degrees of a Scott set is rich and completely determined.

The existential-universal theory of the degrees represented in an arbitrary Scott set is more complex and not at all understood. Groszek and Slaman [6] state that every Scott set has an element of minimal Turing degree, namely a degree $m$ such that $m$ has no nontrivial element strictly below it. The Friedman-McAllister question is universal-existential about an arbitrary Scott set, for every degree $x$ is there a degree $y$ which is Turing incomparable with $x$. In other words, given a Turing degree $x$, can one use a compactness argument to construct a $y$ which is Turing incomparable with $x$ ?

The typical immediate reaction to the question is that there should always be such a $y$ and that it should be routine to exhibit an $x$-recursive tree such that every infinite path in that tree has Turing degree incomparable with $x$. This is not the case. For example, Kučera [9] showed that there is a Scott set $S$ and a nonrecursive degree $x$ from $S$ such that $x$ is recursive in all complete extensions of Peano Arithmetic that appear in $S$. Building $y$ 's incomparable with that $x$ cannot be accomplished using complete extensions of Peano Arithmetic. Similar obstacles appear when one attempts to find $y$ 's incomparable to $x$ by means of other familiar $\Pi_{1}^{0}$ classes.

Even so, for every Scott set $S$ and every nonrecursive $x$ represented in $S$, there is a $y$ in $S$ which is Turing incomparable with $x$. Given $x$, our construction of $y$ is not uniform. If possible, we find $y$ by taking a sequence which is 1-random relative to $x$. If that fails (the nonuniformity), then we apply recent results in the theory of algorithmic randomness to build a recursive tree whose infinite paths are Turing incomparable with $x$.

1.1.2. Definitions and notation. Our computability-theoretic notation generally follows Soare 22] and Odifreddi 15, 16]. An introduction to algorithmic randomness can be found in Li and Vitányi [13. A short survey of it is also given in Ambos-Spies and Kučera 11. Much deeper insight into the subject of algorithmic randomness can be found in a forthcoming book of Downey and Hirschfeldt [4]; a good survey is also in Downey, Hirschfeldt, Nies and Terwijn [5].

We refer to the elements of $2^{\omega}$ as sets or infinite binary sequences. We denote the collection of strings, i.e. finite initial segments of sets, by $2^{<\omega}$. The length of a string $\sigma$ is denoted by $|\sigma|$; for a set $X$, we denote the string consisting of the first $n$ bits of $X$ by $X\lceil n$ and we use similar notation $\sigma\lceil n$ for strings $\sigma$ of length $\geq n$. We let $\sigma * \tau$ denote the concatenation of $\sigma$ and $\tau$ and let $\sigma * Y$ denote the concatenation of $\sigma$ and (the infinite binary sequence) $Y$. We write $\sigma \prec X$ to indicate $X\lceil|\sigma|=\sigma$. If $\sigma \in 2^{<\omega}$, then $[\sigma]$ denotes $\left\{X \in 2^{\omega}: \sigma \prec X\right\}$. 
A $\Sigma_{1}^{0}$ class is a collection of sets that can be effectively enumerated. Such a class can be represented as $\bigcup_{\sigma \in W}[\sigma]$ for some (prefix-free) recursively enumerable (r.e.) set of strings $W$. The complements of $\Sigma_{1}^{0}$ classes are called $\Pi_{1}^{0}$ classes. Any $\Pi_{1}^{0}$ class can be represented by the class of all infinite paths through some recursive tree. We use also relativized versions, i.e., $\Sigma_{1}^{0, X}$ classes and $\Pi_{1}^{0, X}$ classes. $\Pi_{1}^{0}$ classes play an important role in logic, in subsystems of second-order arithmetic, and also in algorithmic randomness. By the relativized tree representation of $\Pi_{1}^{0}$ classes, if $\mathcal{F}$ is a Scott set, $X \in \mathcal{F}$, and $P$ is a nonempty $\Pi_{1}^{0, X}$ class, then $\mathcal{F}$ includes an element of $P$.

Definition 1.2. Let $X$ be a set. A Martin-Löf test relative to $X$ is a uniformly r.e. in $X$ sequence of $\Sigma_{1}^{0, X}$ classes $\left\{U_{n}^{X}\right\}$ such that $\mu\left(U_{n}^{X}\right) \leq 2^{-n}$, where $\mu$ denotes the standard measure on $2^{\omega}$. Then any subclass of $\bigcap_{n \in \omega} U_{n}^{X}$ is called a Martin-Löf null set relative to $X$. If $X=\emptyset$, we simply speak of the Martin-Löf test and Martin-Löf null set. A set $R$ is Martin-Löf random relative to $X$, or 1-random relative to $X$, if $\{R\}$ is not Martin-Löf null relative to $X$. If $X=\emptyset$, we speak of 1-randomness.

Martin-Löf proved that there is a universal Martin-Löf test, $\left\{U_{n}\right\}$, such that for all $R, R$ is 1-random if and only if $R \notin \bigcap_{n \in \omega} U_{n}$. Similarly, there is a universal Martin-Löf test relative to $X,\left\{U_{n}^{X}\right\}$ (uniformly in $X$ ).

We will use $K$ to denote prefix-free Kolmogorov complexity. See Li and Vitányi 13 for details. The version of Kolmogorov complexity relativized to a set $X$ is denoted by $K^{X}$. Schnorr [19] proved that a set is 1-random if and only if for all $n, K(A\lceil n) \geq n+O(1)$. There are several notions of computational weakness related to 1-randomness. They are summarized in the following definition.

Definition 1.3. (1) $\mathcal{L}$ denotes the class of sets which are low for 1-randomness, i.e., sets $A$ such that every 1-random set is also 1-random relative to $A$.

(2) $\mathcal{K}$ denotes the class of $K$-trivial sets, i.e., the class of sets $A$ such that for all $n, K\left(A\lceil n) \leq K\left(0^{n}\right)+O(1)\right.$, where $0^{n}$ denotes the string of $n$ zeros.

(3) $\mathcal{M}$ denotes the class of sets that are low for $K$, i.e., sets $A$ such that for all $\sigma, K(\sigma) \leq K^{A}(\sigma)+O(1)$.

(4) A set $A$ is a basis for 1 -randomness if $A \leq_{T} Z$ for some $Z$ such that $Z$ is 1 -random relative to $A$. The collection of such sets is denoted by $\mathcal{B}$.

Nies [14] proved that $\mathcal{L}=\mathcal{M}$, Hirschfeldt and Nies, see [14, proved that $\mathcal{K}=$ $\mathcal{M}$, and Hirschfeldt, Nies and Stephan $[7$ proved that $\mathcal{B}=\mathcal{K}$. Thus, all these four classes are equal and we have, remarkably, four different characterizations of the same class. That is, $\mathcal{L}=\mathcal{K}=\mathcal{M}=\mathcal{B}$.

Chaitin 3 proved that $K$-trivial sets are $\Delta_{2}^{0}$. Furthermore, by a result of Kučera [10] low for 1-random sets are $G L_{1}$ and, thus, all these sets are, in fact, low. The lowness of these sets also follows from some recent results on this class of sets; see [14.

\section{The MAIN RESUlT}

In this section, we present a solution to the Friedman-McAllister question.

Theorem 2.1. For any Scott set $\mathcal{S}$ and any nonrecursive set $X \in \mathcal{S}$, there is a $Y \in \mathcal{S}$ such that $X$ and $Y$ are Turing incomparable.

Theorem 2.1 is a consequence of the stronger Claim 2.2 
Claim 2.2. For every nonrecursive set $X$ there is a nonempty $\Pi_{1}^{0, X}$ class $\mathcal{P} \subseteq 2^{\omega}$ such that every element of $\mathcal{P}$ is Turing incomparable with $X$.

As we will see, we can do even better for $K$-trivial sets $X$. Namely, we can replace the $\Pi_{1}^{0, X}$ class mentioned in the claim by a (nonrelativized) $\Pi_{1}^{0}$ class.

Proof of Claim 2.2. We split the proof of the claim into two cases.

Case 1. $X$ is not a basis for 1-randomness.

Let $T_{1}$ be a tree recursive in $X$ such that any infinite path in $T_{1}$ is 1-random relative to $X$. We can take, e.g., a tree recursive in $X$ for which the collection of all infinite paths is the $\Pi_{1}^{0, X}$ class which is the complement of $U_{0}^{X}$, the first class appearing in a universal Martin-Löf test relative to $X$. Clearly, any infinite path through $T_{1}$ is Turing incomparable with $X$.

Case 2. $X$ is a basis for 1-randomness.

As we described above, such an $X$ is $K$-trivial, low for 1-randomness, low for $K$, and $\Delta_{2}^{0}$. We use these properties to construct a recursive tree $T_{2}$ such that any infinite path through $T_{2}$ is Turing incomparable with $X$.

Also we not only avoid a lower cone of sets recursive in $X$, but even avoid the lower cone below every set, which is a base for 1-randomness. Since the class of these sets is closed downwards, we obviously get a stronger property.

Since the class $\mathcal{B}$ is equal to $\mathcal{L}$ and also to $\mathcal{M}$, we can equivalently work with any of these characterizations.

Thus, to handle Case 2, it is sufficient to prove the following lemma.

Lemma 2.3. Let $X$ be a nonrecursive $\Delta_{2}^{0}$ set. Then there is a recursive tree $T_{2}$ such that for any infinite path $Y$ in $T_{2}$ we have $Y \Varangle_{T} X$ and $Y$ is not low for 1-randomness (and, thus, $Y \mathbb{Z}_{T} X$ if $X \in \mathcal{L}$ ).

Proof of Lemma 2.3. The proof is by a finite injury priority argument. We build the tree $T_{2}$ by stages. At stage $s+1$, we terminate a string by not extending it to any string of length $s+1$ in $T_{2}$.

We will describe the strategies and leave the rest to the reader.

The strategies have the following general pattern. Each strategy starts to work at a given string $\sigma \in T_{2}$, it acts only finitely often, and it yields as its outcome a nonempty finite collection $Q$ consisting of strings of the same length. Some strategies (called $\mathcal{L}$-strategies) and their outcomes depend not only on $\sigma$ itself, but also on how $\sigma$ arises as a concatenation of strings belonging to outcomes of previous strategies. Furthermore, for each $\alpha \in Q$, the string $\sigma * \alpha$ together with a recursive tree of all strings extending $\sigma * \alpha$ are left for the next strategy. By producing its outcome, each strategy satisfies some particular requirement as explained later.

Avoiding an upper cone above $X$. Let $\sigma \in T_{2}$ be given. Suppose that $\Phi$ is a recursive Turing functional. We act to ensure that for every infinite path $A$ in $T_{2}$, if $A$ extends $\sigma$, then $\Phi(A) \neq X$.

We use the fact that $X$ is $\Delta_{2}^{0}$ to adapt the Sacks Preservation Strategy [18. We monitor the maximum length of agreement between $\Phi(\tau)$, for $\tau$ extending $\sigma$, and the current approximation to $X$. If at stage $s+1$, we see a string $\eta$ of length $s$ on $T_{2}$ for which this maximum has gone higher than ever before, we take the least such $\eta$ and we terminate all extensions of $\sigma$ except for $\eta$. 
If this were to occur infinitely often above $\sigma$, then $X$ would be recursive. Compute $X(n)$ by finding the first stage where the maximum length of agreement between $\Phi(\tau)$, for some $\tau$ extending $\sigma$, and the approximation to $X$ was greater than $n$. Since the length of agreement increases infinitely often, the approximation to $X$ returns to this value infinitely often. But then, since the approximation converges to the value of $X$, the value at the stage we found must be the true value. This is a contradiction to $X$ 's being nonrecursive.

So, this strategy acts finitely often and satisfies the requirement.

Observe that that the strategy yields as its output just one string $\alpha$, where $\alpha$ is the string at which we last terminate all extensions of $\sigma$ that are incompatible with $\alpha$. Otherwise, $\alpha$ is the empty string, if we never do so.

Avoiding the class of sets low for 1-randomness. We will refer to our strategies to avoid the class of sets low for 1-randomness as $\mathcal{L}$-strategies. We will begin by explaining the general idea behind these strategies.

Recall that a set $X$ is low for 1-randomness if and only if every 1-random set $Z$ is also 1-random relative to $X$. In the case that $X$ is low for 1-randomness, we can ensure that $X$ does not compute any path in $T_{2}$ by ensuring that each path $Y$ in $T_{2}$ is not low for 1-randomness, i.e. $Y \notin \mathcal{L}$.

We will ensure that a path $Y$ in $T_{2}$ is not in $\mathcal{L}$ by embedding large intervals of some 1-random set $Z$ into it. In this way, we can recover the 1-random set $Z$ recursively from $Y$ and $\emptyset^{\prime}$ and ensure that $Y$ can enumerate a Martin-Löf test (relative to $Y$ ), which shows that $Z$ is not 1-random in $Y$.

An infinite path $Y$ in $T_{2}$ can be viewed as an infinite concatenation of strings $\alpha_{0} * \alpha_{1} * \alpha_{2} * \cdots$, where each $\alpha_{i}$ is that uniquely determined string compatible with $Y$ that belongs to the outcome of a strategy that started at $\alpha_{0} * \cdots * \alpha_{i-1}$ (where $\alpha_{-1}$ is the empty string). Let us note that due to a standard finite injury priority argument, such a sequence $\left\{\alpha_{i}\right\}_{i \in \omega}$ can be found recursively in $\emptyset^{\prime}$ and $Y$. Then let $Z_{Y}$ denote the set obtained as an infinite concatenation of strings $\alpha_{i_{0}} * \alpha_{i_{1}} * \alpha_{i_{2}} * \cdots$, where $\left\{i_{j}\right\}_{j \in \omega}$ is a recursive increasing sequence of indices of those strings $\alpha_{i}$ that belong to outcomes of $\mathcal{L}$-strategies, i.e., of those $i$ 's for which a strategy that started at $\alpha_{0} * \alpha_{1} * \cdots * \alpha_{i-1}$ was an $\mathcal{L}$-strategy. The general goal of $\mathcal{L}$-strategies is to ensure that for any infinite path $Y$ in $T_{2}, Z_{Y}$ is 1-random but is not 1-random relative to $Y$.

To guarantee that $Z_{Y}$ is not 1-random in $Y$, we have to satisfy for all $e$ the requirement $Z_{Y} \in U_{e}^{Y}$, where $\left\{U_{e}^{Y}\right\}_{e \in \omega}$ is a universal Martin-Löf test relative to $Y$ (uniformly in $Y$ ). As is standard, we may let $U_{e}^{Y}$ be $\bigcup_{k} V_{e+k+1}^{k, Y}$, where $\left\{V_{i}^{k, Y}\right\}_{i, k \in \omega}$ is a uniformly r.e. in $Y$ sequence of all Martin-Löf tests relative to $Y$ (uniformly in $Y)$, and $\left\{V_{i}^{k, Y}\right\}_{i \in \omega}$ is the $k$-th test.

To guarantee that $Z_{Y}$ is 1-random, we fix a $\Pi_{1}^{0}$ class $\bar{U}_{0}$ of 1-random sequences and fix a recursive tree $T^{*}$ such that the infinite paths in $T^{*}$ are exactly the members of $\bar{U}_{0}$. We will ensure that each initial segment of $Z_{Y}$ extends to an element of $\bar{U}_{0}$.

Suppose now, that $\sigma \in T_{2}$ and $e$ are given, and let $\alpha_{0}, \ldots, \alpha_{k}$ be the strings for which $\sigma=\alpha_{0} * \alpha_{1} * \cdots * \alpha_{k}$, where each $\alpha_{i}$ belongs to an outcome of the strategy that started at $\alpha_{0} * \alpha_{1} * \cdots * \alpha_{i-1}$. Let $\tau_{\sigma}$ be the string $\alpha_{i_{0}} * \alpha_{i_{1}} * \cdots * \alpha_{i_{j}}$, where $i_{0}, i_{1}, \ldots, i_{j}$ are indices (in increasing order) of those $\alpha_{i}$ 's that belong to outcomes of $\mathcal{L}$-strategies, i.e., of those $i$ 's such that the strategy that started at $\alpha_{0} * \alpha_{1} * \cdots * \alpha_{i-1}$ was an $\mathcal{L}$-strategy. Roughly speaking, $\tau_{\sigma}$ is the finite sequence already embedded into $\sigma$ which can be extended to an infinite path in $T^{*}$. 
Observe that for any set $Z$ the set $\tau_{\sigma} * Z$ is not 1 -random relative to the set $\sigma * Z$. In fact, $\tau_{\sigma} * Z$ is recursive in $\sigma * Z$ as it is obtained by appending all but finitely much of $\sigma * Z$ to $\tau_{\sigma}$. Thus, $\tau_{\sigma} * Z \in U_{e}^{\sigma * Z}$. If we had no other requirements to satisfy, we could restrict the infinite extensions of $\sigma$ in $T_{2}$ to those of the form $\sigma * Z$ for which $\tau_{\sigma} * Z$ is 1 -random (using the $\Pi_{1}^{0}$ class $\bar{U}_{0}$ ). But, of course, we have to satisfy our requirement in a finitary way to leave space for the cone-avoiding strategies. The idea here is to make any infinite path through $T_{2}$ extending $\sigma$ locally 1-random.

Thus, we design our tree so that enough of such a $Z$ is embedded in the extensions of $\sigma$ to ensure that $\left[\tau_{\sigma} *(Z \backslash i)\right] \subseteq U_{e}^{\sigma * Z}$ for some $i$. The crucial thing here is that we can accomplish this objective in a finite way. That is, we can effectively compute (from $\sigma, \tau_{\sigma}$ and $e$ ) an $i$ such that $\left[\tau_{\sigma} *(Z\lceil i)] \subseteq U_{e}^{\sigma * Z}\right.$ for all sets $Z$. Intuitively, for any $Z, \tau_{\sigma} * Z$ is not 1 -random relative to $\sigma * Z$ and we can calculate how long it takes for $\sigma * Z$ to recognize the failure of relative 1-randomness.

We give this calculation in detail. Given $\sigma$ and $\tau_{\sigma}$ find a Martin-Löf test $\left\{B_{j}^{X}\right\}_{j \in \omega}$ relative to $X$ (uniformly in $X$ ) with index $b$ such that $B_{j}^{X}=\left[\left(\tau_{\sigma} * X^{*}\right)\lceil j]\right.$, where $X^{*}$ is the set for which $X=(X \uparrow|\sigma|) * X^{*}$. Then we obviously have $B_{j}^{\sigma * Z}=\left[\left(\tau_{\sigma} * Z\right)\lceil j]\right.$, for any set $Z$. By the construction of $\left\{U_{e}^{X}\right\}_{e \in \omega}$, the universal Martin-Löf test (relative to $X$ ), and since $b$ is an index of the test $\left\{B_{j}^{X}\right\}_{j \in \omega}$, we have $B_{e+b+1}^{\sigma * Z} \subseteq U_{e}^{\sigma * Z}$ for all sets $Z$. It follows that $\left[\tau_{\sigma} *(Z\lceil i)] \subseteq U_{e}^{\sigma * Z}\right.$ for all sets $Z$ and $i$ such that $\left|\tau_{\sigma}\right|+i \geq e+b+1$. Our calculation chooses the least such $i$.

It only remains to put a restriction on $T_{2}$ to ensure that $\sigma * \alpha$ is extendable to an infinite path in $T_{2}$ for strings $\alpha$ of length $i$ if and only if $\tau_{\sigma} * \alpha$ is extendable to an infinite path in $T^{*}$, the recursive tree whose infinite paths are exactly the elements of $\bar{U}_{0}$ and hence are 1-random.

The strategy, given $\sigma \in T_{2}$ and $e$, where $\sigma=\alpha_{0} * \alpha_{1} * \cdots * \alpha_{k}$ with properties of $\alpha_{i}$ 's described above, is precisely as follows. Find the corresponding string $\tau_{\sigma}$, then compute an $i$ such that $\left[\tau_{\sigma} *(Z\lceil i)] \subseteq U_{e}^{\sigma * Z}\right.$ for all sets $Z$. Now for each $\beta$ such that $|\beta| \geq i$, we terminate the string $\sigma * \beta$ in $T_{2}$ if $\tau_{\sigma} *(\beta\lceil i)$ is not extendable to a string of length $|\sigma * \beta|$ in the recursive tree $T^{*}$ which represents the $\Pi_{1}^{0}$ class $\bar{U}_{0}$. This strategy acts only finitely often and eventually reaches its goal. Observe that the strategy yields a finite collection of strings of the same length $Q$, where all requests on $Q$ are satisfied.

With Lemma 2.3, we have completed the proof of Claim 2.2.

2.1. An $\mathcal{M}$ variation. Since $\mathcal{L}$ and $\mathcal{M}$ are equal, we can equivalently use the characterization of $\mathcal{M}$ to design our strategy to handle Case 2, namely, our strategy for avoiding the class of sets which are bases for 1-randomness. For the convenience of the reader we present also a variant of a strategy expressed in terms of $\mathcal{M}$.

Given a $\sigma$, we want to ensure that each infinite path $Y$ extending $\sigma$ in $T_{2}$ can give a shorter description of some string $\tau$ than any description possible without $Y$. Let $c$ be the amount that we want to shorten the description. We will compute an $m$ (see below), and we want to ensure

$$
K\left(Y\lceil m)-c \geq K^{Y}(Y \uparrow m) .\right.
$$

We choose $m$ much larger than $K(\sigma)$ and $c$. The maximum of $2^{|\sigma|+K(\sigma)+c+d}$ and 10 is large enough (where a constant $d$ is explained below). For each string $\tau$ extending $\sigma$ of length $m$ let $\tau^{*}$ denote the string of length $m-|\sigma|$ for which $\tau=\sigma * \tau^{*}$. It is easy to see that $K^{Y}(Y \uparrow m)$ is less than or equal to $2 \log (m)$, 
since $Y$ can describe its first $m$ values using the description of $m$. (As a caveat, this bound may only apply to sufficiently large $m$ because of the fixed cost of interpreting binary representations. This is fixed data and we can assume that $m$ is large enough for the upper bound to apply.)

On the other hand, $K\left(\tau^{*}\right) \leq K(\tau)+K(\sigma)+d$ for some constant $d$ independent of a choice of $\tau$. By terminating strings with shorter descriptions, we can ensure that for each $Y$ extending $\sigma$ in $T_{2}$, the $\tau^{*}$ of $\tau=Y \uparrow m$ satisfies $K\left(\tau^{*}\right) \geq m-|\sigma|$. This is similar to making a recursive tree of 1-random sets, but here we are making any path in $T_{2}$ (merely) locally 1-random to ensure that infinite paths in $T_{2}$ are not low for $K$.

We can now calculate:

$$
K(\tau) \geq K\left(\tau^{*}\right)-K(\sigma)-d
$$

and substituting for $K\left(\tau^{*}\right)$,

$$
K(\tau) \geq(m-|\sigma|)-K(\sigma)-d .
$$

Since $K^{Y}(Y \uparrow m) \leq 2 \log (m)$, it is sufficient to ensure that

$$
m-|\sigma|-K(\sigma)-d-c \geq 2 \log (m),
$$

or, equivalently,

$$
m \geq 2 \log (m)+|\sigma|+K(\sigma)+c+d .
$$

If $m \geq 2^{|\sigma|+K(\sigma)+c+d}$, then it is sufficient to ensure $m \geq 3 \log (m)$. This holds if $m$ is greater than 10 .

So, the strategy working above $\sigma$ to ensure that $Y$ is not low for $K$ reserves the collection of extensions of $\sigma$ of length $m$, and at most half of them are eventually stopped to be extendable to an infinite path in $T_{2}$. So, it satisfies its requirement and acts only finitely often.

Remark. We have not addressed the question whether it is provable in the subsystem of second-order arithmetic $W K L_{0}$ that for every nonrecursive set $X$ there is a nonrecursive set $Y$ which is Turing incomparable with $X$ (see Problem 3.2. part 1 in Cenzer and Jockusch [2]).

\section{AN OPEN PROBLEM}

Suppose that $\mathcal{F}$ is a Scott set and let $\mathcal{D}^{\mathcal{F}}$ denote the partial order of the Turing degrees which are represented by elements of $\mathcal{F}$. According to Theorem 2.1

$$
\mathcal{D}^{\mathcal{F}}=\forall d>0 \exists x\left(d \geq_{T} x \text { and } x \nsupseteq_{T} d\right) .
$$

The dual theorem of Groszek and Slaman [6] states that every Scott set has an element of minimal Turing degree:

$$
\mathcal{D}^{\mathcal{F}} \models \exists d>0 \forall x \neg\left(d>_{T} x \text { and } x>_{T} 0\right) .
$$

Together, these results are sufficient to determine for any sentence in the language of partial orders of the form $\forall d \exists x \varphi(d, x)$, where $\varphi$ is quantifier-free, whether that sentence holds in $\mathcal{D}^{\mathcal{F}}$. Furthermore, such sentences hold in $\mathcal{D}^{\mathcal{F}}$ if and only if they hold in $\mathcal{D}$, the Turing degrees of all sets.

By Lerman 12 and Shore [21, the general $\forall \exists$-theory of $\mathcal{D}$ is decidable. The proof of decidability rests on two technical results. The first is a general extension theorem due to Sacks [17] which, like Theorem 2.1, constructs degrees $x$ incomparable to or above given ones $d$. The second is Lerman's [1] theorem that every finite lattice 
is isomorphic to an initial segment of the Turing degrees which, like Formula (11), produces degrees $d$ which limit the possible types of $x$ 's which are below $d$.

Superficially, Theorem 2.1 and the existence of minimal degrees suggest that the $\forall \exists$-theory of $\mathcal{D}^{\mathcal{F}}$ resembles that of $\mathcal{D}$. However, the actual proofs are quite different, and we are left with the following question.

Question 3.1. Suppose that $\mathcal{F}$ is a $\operatorname{Scott}$ set. Is $\mathcal{D}^{\mathcal{F}} \forall \exists$-elementarily equivalent to $\mathcal{D}$ ?

\section{REFERENCES}

[1] Klaus Ambos-Spies and Antonín Kučera. Randomness in computability theory. In Computability theory and its applications (Boulder, CO, 1999), volume 257 of Contemp. Math., pages 1-14. Amer. Math. Soc., Providence, RI, 2000. MR1770730 (2001d:03112)

[2] Douglas Cenzer and Carl G. Jockusch, Jr. $\Pi_{1}^{0}$ classes - structure and applications. In Computability theory and its applications (Boulder, CO, 1999), volume 257 of Contemp. Math., pages 39-59. Amer. Math. Soc., Providence, RI, 2000. MR1770733 (2001h:03074)

[3] G. J. Chaitin. Algorithmic information theory. IBM J. Res. Develop., 21:350-359, 496, 1977. MR0456932 (56:15151)

[4] R. Downey and D. R. Hirschfeldt. Algorithmic randomness and complexity. to appear.

[5] R. Downey, D. R. Hirschfeldt, A. Nies, and S. A. Terwijn. Calibrating randomness. Bull. Symbolic Logic 12:411-491 (2006). MR 2248591

[6] Marcia J. Groszek and Theodore A. Slaman. $\Pi_{1}^{0}$ classes and minimal degrees. Ann. Pure Appl. Logic, 87(2):117-144, 1997. Logic Colloquium '95 Haifa. MR1490050 (99f:03058)

[7] D. R. Hirschfeldt, A. Nies, and F. Stephan. Using random sets as oracles. to appear.

[8] Carl G. Jockusch, Jr. and Robert I. Soare. $\Pi_{1}^{0}$ classes and degrees of theories. Trans. Amer. Math. Soc., 173:33-56, 1972. MR0316227(47:4775)

[9] Antonín Kučera. On the role of $\mathbf{0}^{\prime}$ in recursion theory. In Logic colloquium '86 (Hull, 1986), volume 124 of Stud. Logic Found. Math., pages 133-141. North-Holland, Amsterdam, 1988. MR.922107 (89b:03070)

[10] Antonín Kučera. On relative randomness. Ann. Pure Appl. Logic, 63(1):61-67, 1993. 9th International Congress of Logic, Methodology and Philosophy of Science (Uppsala, 1991). MR.1238109 (95c:03103)

[11] M. Lerman. Initial segments of the degrees of unsolvability. Ann. of Math., 93:311-389, 1971. MR0307893 (46:7008)

[12] M. Lerman. Degrees of Unsolvability. Perspectives in Mathematical Logic. Springer-Verlag, Heidelberg, 1983. 307 pages. MR708718 (85h:03044)

[13] Ming Li and Paul Vitányi. An introduction to Kolmogorov complexity and its applications. Graduate Texts in Computer Science. Springer-Verlag, New York, second edition, 1997. MR:1438307 (97k:68086)

[14] André Nies. Lowness properties and randomness. Adv. Math., 197(1):274-305, 2005. MR2166184 (2006j:68052)

[15] Piergiorgio Odifreddi. Classical recursion theory, volume 125 of Studies in Logic and the Foundations of Mathematics. North-Holland Publishing Co., Amsterdam, 1989. The theory of functions and sets of natural numbers, With a foreword by G. E. Sacks. MR982269 (90d:03072)

[16] Piergiorgio Odifreddi. Classical recursion theory. Vol. II, volume 143 of Studies in Logic and the Foundations of Mathematics. North-Holland Publishing Co., Amsterdam, 1999. MR.982269 (90d:03072)

[17] Gerald E. Sacks. On suborderings of degrees of recursive unsolvability. Z. Math. Logik Grundlag. Math., 17:46-56, 1961. MR0131973(24:A1820)

[18] Gerald E. Sacks. On the degrees less than $\mathbf{0}^{\prime}$. Ann. of Math., 77:211-231, 1963. MR0146078 (26:3604)

[19] C.-P. Schnorr. A unified approach to the definition of random sequences. Math. Systems Theory, 5:246-258, 1971. MR0354328 (50:6808)

[20] Dana Scott. Algebras of sets binumerable in complete extensions of arithmetic. In Recursive Function Theory, volume 5 of Proceedings of Symposia in Pure Mathematics, pages 117-121, American Mathematical Society, Providence, R.I., 1962. MR0141595 (25:4993) 
[21] Richard A. Shore. On the $\forall \exists$-sentences of $\alpha$-recursion theory. In R. O. Gandy, J. Fenstad and G. E. Sacks, editors, Generalized Recursion Theory II, volume 94 of Stud. Logic Foundations Math., pages 331-354, Amsterdam, 1978. North-Holland Publishing Co. MR516943 (80e:03055)

[22] Robert I. Soare. Recursively Enumerable Sets and Degrees. Perspectives in Mathematical Logic, Omega Series. Springer-Verlag, Heidelberg, 1987. MR882921 (88m:03003)

Department of Theoretical Computer Science and Mathematical logic, Faculty of Mathematics and Physics, Charles University, Malostranské nám. 25, 11800 Praha 1, Czech Republic

E-mail address: kucera@ksi.mff.cuni.cz

Department of Mathematics, The University of California, Berkeley, Berkeley, CALifornia 94720-3840

E-mail address: slaman@math.berkeley.edu 